\section{Claude Marineau Philippe Mérel Guy A. Rouleau Gilles Thomas}

\title{
Le gène de la neurofibromatose de type 2
}

La neurofibromatose de type 2 (NF2) est une maladie héréditaire prédisposant, principalement, au développement de schwannomes vestibulaires et de méningiomes. Bien que cette affection soit une maladie relativement rare, les schwannomes et les méningiomes, que l'on retrouve sous forme sporadique, représentent une proportion importante des tumeurs cérébrales. Une stratégie de clonage positionnel a permis d'identifier, sur le chromosome 22, un gène vraisemblablement déficient chez la plupart des malades atteints de NF2. Ce gène code pour une protéine de $66 \mathrm{kDa}$, nommée schwannomine ou merline, ayant une forte analogie de séquence avec les protéines de la famille ezrine-radixine-moésine (ERM). La schwannomine pourrait donc assurer le maintien d'interactions entre la membrane et le cytosquelette. Les études cytogénétiques des tumeurs, de même que les mutations observées dans le génome de cellules tumorales, suggèrent que la schwannomine joue un rôle de suppresseur de tumeur.

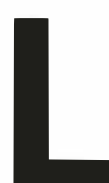

es neurofibromatoses sont des maladies à transmission autosomique dominante prédisposant au développement de tumeurs du système nerveux. La neurofibromatose de type 1 $(\mathrm{NF} 1)$, encore appelée neurofibromatose périphérique ou maladie de von Recklinghausen, est parmi les maladies génétiques les plus fréquentes (1/3000 nouvelles naissances). Elle se caractérise par la présence de taches café au lait, le développement de neurofibromes périphériques et, dans la grande majorité des cas, par la présence de nodules de Lisch (Tableau I) [1, 2]. Plus rarement, la NF1 peut être associée à des anomalies osseuses et à l'apparition de gliomes du nerf optique [1].
La gravité de la maladie est très variable d'un sujet à un autre, même lorsque ces sujets appartiennent à la même famille. La neurofibromatose de type 2 (NF2) est dix fois plus rare et ses manifestations cliniques sont beaucoup moins variables. Les premiers signes de la maladie apparaissent généralement durant l'adolescence ou au début de l'âge adulte. La NF2 prédispose au développement de schwannomes, surtout sur la branche vestibulaire du huitième nerf crânien (neurinomes acoustiques), de méningiomes et d'épendymomes. La présence d'une cataracte juvénile, la seule manif estation non tumorale de la NF2, est observée chez près de $40 \%$ des sujets (Tableau I) [1, 2]. La présence de schwan- 


\section{RÉFÉRENCES}

1. Huson SM, Hughes RAC. The neurofibromatosis, a pathogenetic and clinical overview. London : Chapman and Hall Medical, 1994.

2. National Institutes of Health. Neurofibromatosis Conference Statement. National Institutes of Health Consensus Development Conference Statement. Neurofibromatosis $1988 ; 1: 172-8$.

3. Barker D, Wright E, Nguyen K, et al. Gene for von Recklinghausen neurofibromatosis is in the pericentromeric region of chromosome 17. Science $1987 ; 236: 1100-2$

4. Seizinger BR, Rouleau GA, Ozelius LJ, et al. Genetic linkage of von Recklinghausen neurofibromatosis to the nerve growth receptor gene. Cell $1987 ; 49: 589-94$.

5. Rouleau GA, Wertelecki W, Haines JL, et al. Genetic linkage of bilateral acoustic neurofibromatosis to a DNA marker on chromosome 22 . Nature $1987 ; 329: 246-8$.

6. Viskochil D, Buchberg AM, Xu G, et al. Deletions and a translocation interrupt a cloned gene at the neurofibromatosis type 1 locus. Cell $1990 ; 62: 187-92$

7. Rouleau GA, Mérel P, Lutchman M, et al. Alteration in a new gene encoding a putative membrane organizing protein causes neurofibromatosis type 2 . Nature $1993 ; 363$ : 515-21.

8. Trofatter JA, Mac Collin M, Rutter JL, $e t$ al. A novel moesin-, ezrin-, radixin-like gene is a candidate for the neurofibromatosis 2 tumor suppressor. Cell $1993 ; 72: 791-800$.

9. Butler J, Brooks R, Netsky D. Classification and biology of brain tumours. In: Youmans JR, ed. Neurological surgery. Philadelphia : W.B. Saunders, 1982.

10. Zang KD, Singer H. Chromosomal constitution of meningiomas. Nature 1967 . $216: 84-5$.

11. Zang KD. Cytological and cytogenetical studies on human meningioma. Cancer $G e$ net Cytogenet $1982 ; 6: 249-75$. nomes vestibulaires bilatéraux est la principale caractéristique de la NF2 et est suffisante pour le diagnostic de la maladie [2]. En absence de traitement, le développement de ces tumeurs produit des compressions nerveuses conduisant à des diminutions de l'acuité auditive allant jusqu'à la surdité, à des troubles de l'équilibre et à des paralysies faciales. Les symptômes de la NF2, qui sont généralement plus sévères que ceux de la NF1, peuvent mettre en jeu le pronostic vital.

L'existence de symptômes communs aux deux types de neurofibromatose, NF1 et NF2, complique le diagnostic clinique et a longtemps été une source de confusion. Par exemple, les taches café au lait sont aussi observées chez des patients NF2 mais en moins grand nombre que chez les patients NF1, la différence étant plus quantitative que qualitative. Il en est de même pour les tumeurs périphériques que l'on retrouve dans les deux types de neurofibromatose. Il n'existait, jusqu'en 1987, aucune preuve démontrant que ces deux maladies soient génétiquement différentes ni qu'elles puissent résulter de mutations spécifiques d'un même gène. La liaison génétique de la NF1 au chromosome 17 et celle de la NF2 au chromosome 22 ont permis de répondre à cette question [3-5]. Le gène de la NF1 a été identifié en 1990 ; il code pour une protéine appelée neurofibromine intervenant dans le contrôle de l'activité GTPasique des protéines oncogéniques Ras et pourrait avoir des propriétés "suppresseur de tumeur" [6]. Le gène de la neurofibromatose de type 2 a été identifié plus récemment grâce à une stratégie de clonage positionnel $\left(m / s n^{\circ} 4\right.$, vol. $\left.9, p .484,[7,8]\right)$. Sa localisation résulte d'observations

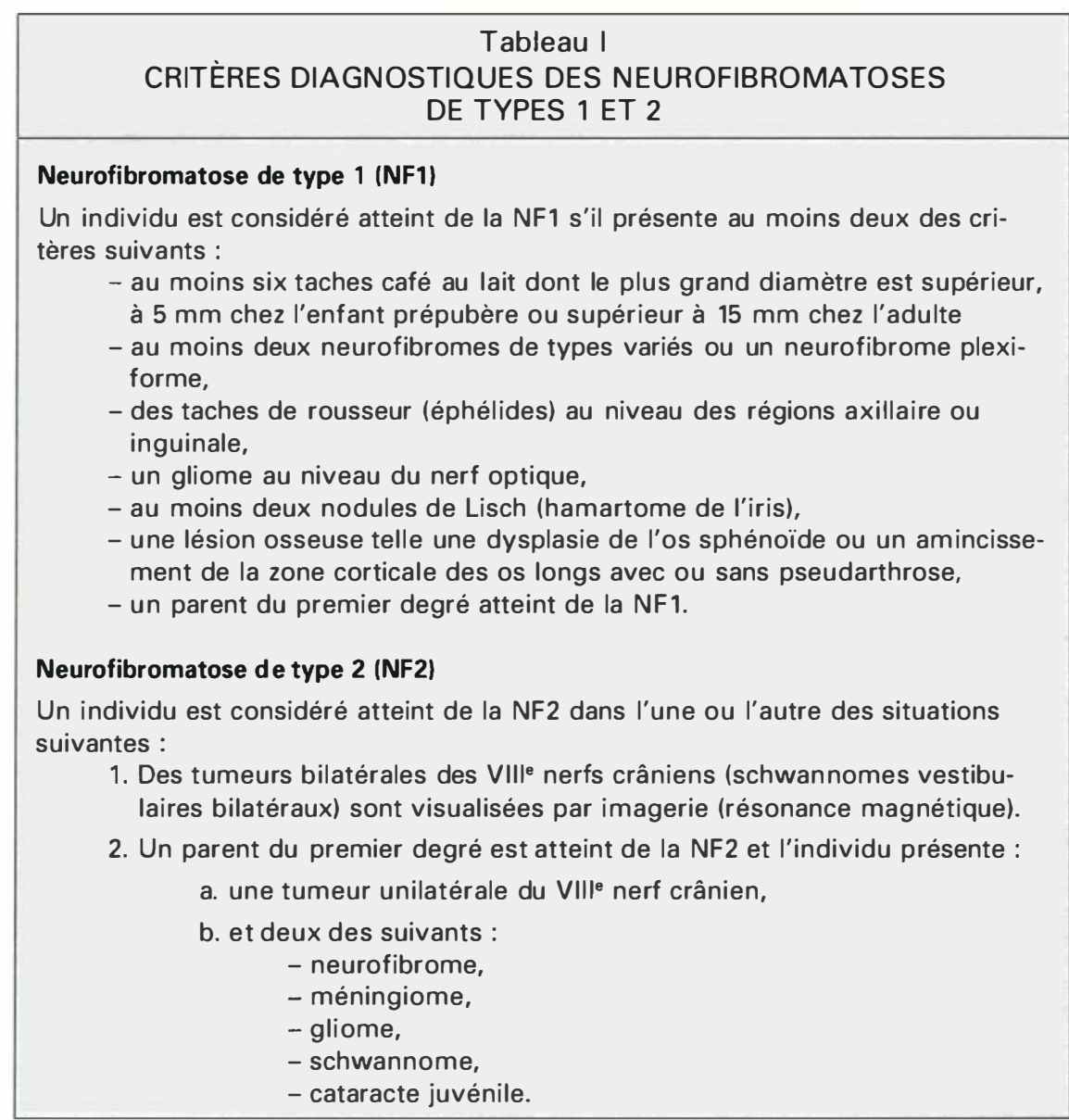

N.B. Ces critères ont été définis par un groupe d'experts réunis par le National Institute of Health pour identifier avec certitude les individus atteints dans le but de réaliser des recherches cliniques et de liaison génétique. Des situations plus complexes peuvent être observées dans la pratique médicale [2]. 
cytogénétiques réalisées sur des tumeurs et d'études de coségrégation dans les familles atteintes de la NF2. Le clonage a nécessité une cartographie physique précise de la région chromosomique impliquée et une identification systématique des séquences codantes $\left(\mathrm{m} / \mathrm{s} n^{\circ} 4\right.$, vol. 9, $p$. 484).

\section{Méningiomes et schwannomes}

Les méningiomes et les schwannomes constituent à eux seuls environ $30 \%$ des tumeurs primaires du système nerveux, ce qui représenterait, en France, plus de 3000 cas annuels [9]. Ces tumeurs se développent la plupart du temps après 50 ans dans la population générale. Les sujets atteints de NF2 représentent environ $5 \%$ de ces cas, et les tumeurs apparaissent en moyenne 30 ans avant celles de la population générale.

Les méningiomes sont des tumeurs généralement bénignes, issues des cellules arachnoïdes des méninges. Les analyses cytogénétiques des méningiomes, réalisées dès 1967 par Zang et Singer et précisées au début des années 1970 lorsque les techniques de marquage en bande des chromosomes apparurent, ont constitué la première démonstration de la perte récurrente d'un chromosome spécifique dans un type tumoral déterminé $[10,11]$. Ces travaux démontrèrent une fréquente monosomie du chromosome 22 dans les méningiomes. Ces pertes somatiques de matériel génétique furent mieux précisées au cours des années 1980 par la démonstration, chez des sujets hétérozygotes, de pertes d'allèles portés par le chromosome 22 dans l'ADN extrait de méningiomes [12].

Les schwannomes sont des tumeurs bénignes qui, comme leur nom l'indique, se développent à partir des cellules de Schwann. Ils sont particulièrement fréquents au contact de la branche vestibulaire de la huitième paire de nerfs crâniens. En 1986, un groupe de chercheurs démontrait l'existence de pertes de matériel génétique sur le chromosome 22 dans $44 \%$ des schwannomes sporadiques étudiés [13]. Aucune perte allélique n'était observée pour les onze autres chromosomes testés, suggérant ainsi que la perte de matériel sur le chro$\mathrm{m} / \mathrm{s} n^{\circ} 1$, vol. 11 , janvier 95 mosome 22 était un événement spécifique. De plus, des pertes alléliques sur le chromosome 22 furent ultérieurement observées dans des schwannomes d'origines diverses, c'est-à-dire non vestibulaires $[14,15]$.

Les études décrites ci-dessus, effectuées sur des tumeurs sporadiques, ont été reprises sur des tumeurs de malades NF2. Seizinger $e t$ al. ont ainsi analysé six tumeurs, soit deux schwannomes vestibulaires, un méningiome et trois schwannomes non vestibulaires [16]. Des pertes alléliques sur le chromosome 22 ont été observées dans cinq de ces six tumeurs, confirmant ainsi l'hypothèse qu'une perte de matériel génétique est à l'origine des schwannomes, tant sporadiques que familiaux. L'ensemble de ces travaux fut réalisé à une époque où l'hypothèse de Knudson, suggérant que la croissance néoplasique puisse résulter de l'inactivation des deux copies cellulaires d'un gène dit "suppresseur de tumeur", venait d'être brillamment démontrée par l'isolement du gène $R b$ impliqué dans le rétinoblastome familial [17, 18]. L'analyse des schwannomes et des méningiomes conduisait donc naturellement à suggérer l'existence chez les sujets atteints de NF2 d'une altération d'un gène suppresseur de tumeur porté par le chromosome 22.

\section{Liaison génétique}

Une famille de taille exceptionnelle, dont vingt-trois membres étaient atteints de la NF2, a permis d'établir que, dans cette famille, le défaut génétique responsable de la NF2 était situé sur le bras long du chromosome 22 [5]. Des études ultérieures, rendues possibles par le recensement de nombreuses familles de taille plus restreinte atteintes de NF2, devaient montrer que les altérations responsables étaient très vraisemblablement toutes localisées dans la même région du génome [19]. La position de cette région a pu être précisée grâce au développement d'un nombre important de marqueurs de polymorphismes du chromosome 22. Elle était située dans la portion médiane du bras long du chromosome 22. L'existence de recombinants rares placèrent successivement le gène NF2 entre les marqueurs I)22S1 et I)22S28 [20] puis entre I)22S212 et
I)22S32 (figures 1 et 2A) ([19, 21] et résultats non publiés).

Ces études eurent des applications pratiques immédiates puisqu'elles permirent de réaliser des diagnostics présymptomatiques dans les cas familiaux de NF2 [21]. Avant l'arrivée d'un test génétique, tous les individus potentiellement à haut risque devaient être suivis régulièrement. Le suivi consiste en un examen de la peau pour la présence de neurofibromes et de schwannomes, un examen audiologique et une imagerie cérébrale par résonance magnétique nucléaire afin de détecter la présence de tumeurs. L'usage d'un test présymptomatique permet maintenant d'identifier les individus qui ne sont pas à risque et leur évite donc le suivi médical. L'identification des individus à risque pourrait permettre une résection précoce des tumeurs, évitant les complications les plus sérieuses associées à la NF2. Il n'existe toutefois encore aucune donnée concernant ce type de suivi et d'intervention chirurgicale. Le test présymptomatique est limité par le fait qu'environ $50 \%$ des cas de NF2 sont dus à de nouvelles mutations. Ces malades ne peuvent donc pas être identifiés avant l'apparition des premiers symptômes.

\section{Cartographie physique}

Tout en sachant que la région entre I)22S212 et I)22S32 était petite, aucune recombinaison génétique n'ayant été observée entre ces marqueurs, il était difficile d'avoir une connaissance précise de sa taille sans réaliser une cartographie physique de la région $(f i-$ gure $2 B$ ). A priori, plusieurs stratégies sont possibles pour établir une telle carte. Cependant, la région comprise entre I)22S212 et I)22S32 est le site du point de cassure d'une translocation chromosomique $t(11 ; 22)$ très fréquemment observée dans un groupe de tumeurs malignes de l'enfant n'ayant pas de rapport avec la NF2, la famille des tumeurs d'Ewing $\left(\mathrm{m} / \mathrm{s} n^{\circ} 9\right.$, vol. $8, p$. 994). Cette observation devait avoir une influence déterminante sur le choix de la stratégie [22]. En effet, dans le but de cloner ce point de cassure chromosomique, plusieurs loci indépendants avaient été localisés au sein de cette région et l'isolement itératif de cosmides chevauchants consti- 


\section{RÉFÉRENCES}

12. Seizinger BR, de la Monte S, Atkins I, et al. Molecular genetic approach to human meningioma : loss of genes on chromosome 22. Proc Nall Acad Sci USA $1987 ; 84$ : 541923.

13. Seizinger BR, Martuza RI, Gusella JF. Loss of genes on chromosome 22 in tumorigenesis of human acoustic neuromas. Nature $1986 ; 332$ : $644-7$

14. Couturier J, Delattre O, Kujas M, et al. Assessment of chromosome 22 anomalies in neurinomas by combined karyotype and RFI.P analyses. Cancer (ienet Cytogenet 199(); $45: 55-62$

15. Fontaine B, Hanson MP, Von Sattel IP, et al. Loss of chromosome 22 alleles in human sporadic spinal schwannomas. Ann Neurol 1991 ; 29 : 183-6.

16. Seizinger BR, Rouleau (;A, Ozelius LJ, et al. Common pathogenetic mechanism for three tumour types in bilateral acoustic neurofibromatosis. Science 1987 ; 236 : 317-9.

17. Knudson AG. Mutation and cancer: a statistical study. Proc Nall Acad Sci USA 1971 ; $68: 82()-3$

18. Friend SH, Bernards HR, Rogelj, et al. A human DNA segment with properties of the gene that predisposes to retinoblastoma and osteosarcoma. Nature $1986 ; 323: 643-6$.

19. Narod SA, Parry DM, Parboosingh J, et al. Neurofibromatosis type 2 appears to be a genetically homogeneous disease. $\mathrm{Am} J$ Hum (ienet $1992 ; 51$ : 486-96.

20. Rouleau (;A, Seizinger BR, Wertelecki $W$, et al. Flanking markers bracket the neurofibromatosis type 2 (NF-2) gene on chromosome 22. Am f Hum (ienel 1990 ; 46 : 3238.

21. Ruttledge MH, Narod SA, Dumanski JP, el al. Presymptomatic diagnosis for neurofibromatosis 2 with chromosome 22 markers. Neurology $1993 ; 43: 1753-6($ ).

22. Delattre O, Zucman J, Plougastel B, et al. Cloning and characterization of the Ewing's sarcoma and peripheral neuroepithelioma $\mathrm{t}(11 ; 22)$ translocation breakpoints. Nalure tuant des contigs avait déjà été entrepris pour certains d'entre eux. Ce début de cartographie physique fut donc poursuivi jusqu'à ce que la région I)22S212 à I)22S32 soit couverte par un ensemble quasi continu de cosmides chevauchants (figures $2 B$ et $2 C$ ). Les régions non recouvertes furent comblées par l'isolement de chromosome artificiel de levure (YAC: yeast artificial chromosome).

Ce travail réalisé, il fut possible de conduire deux séries d'expériences. La première série consista à identifier des fragments de génome conservés à travers la phylogénie et, si possible, dépourvus de séquences répétées et à examiner si ces fragments permettaient de détecter des transcrits sur des répliques de type northern. Plusieurs régions transcrites furent ainsi identifiées ; l'une d'entre elles correspondait à un gène inconnu dont le transcrit mature était de 4,4 kilobases ( $\mathrm{kb}$ ) [7]. La deuxième série d'expériences consista à utiliser ces fragments comme sonde pour rechercher, dans l'ADN extrait de patients atteints de NF2, l'éventuelle présence de fragments d'ADN de taille anormale. Ces expériences furent réalisées en utilisant l'électrophorèse en champs pulsés (PFGE: pulse field gel electrophoresis) et la technique de Southern. Une telle approche conduisit à identifier, au sein d'un groupe de quarante sujets indépendants atteints de NF2, deux malades dont l'ADN présentait une anomalie. L'hybridation in situ sur noyaux interphasiques devait démontrer que ces deux malades présentaient des délétions interstitielles de respectivement 130 et $40 \mathrm{~kb}$ (figure 2C, malades R2399 et R2142) [7]. Ces délétions contenaient donc des fragments conservés à travers la phylogénie et détectant un transcrit de $4,4 \mathrm{~kb}$. Un de ces fragments fut utilisé pour isoler l'ADNc correspondant.

\section{Le gène $\mathrm{SCH}$}

Un clone d'une taille de 2,0 kb fut isolé d'une banque d'ADNc préparée à partir de cerveau foetal humain. La détermination de sa séquence montra qu'il contenait un cadre ouvert de lecture complet de 1785 paires de bases (pb) codant pour une protéine de 595 acides aminés. Pour adopter une nomenclature comparable à celle qui avait été choisie pour la NFl, cette protéine fut appelée schwannomine. Cette protéine de $66 \mathrm{kDa}$ se révéla identique à la merline (MERLIN : moesin-, exrin-, radixin-like protein), une protéine isolée au même moment par un autre groupe qui avait poursuivi une stratégie très proche de celle décrite ici [8]. Le gène codant pour la schwannomine fut appelé $S C H$ par notre groupe; il est divisé en 16 exons couvrant environ 120 kb d'ADN génomique (figure 2I))[7]. Un exon alternatif a été identifié récemment $\left(\mathrm{m} / \mathrm{s} n^{\circ} \mathrm{\sigma}\right.$ 7, vol. 10, p. 729, [23]). Ce nouvel exon est introduit après l'exon 15 et, lorsqu'il est présent dans le transcrit mature, entraîne la synthèse d'une protéine de 590 acides aminés (nommée isoforme II).

Afin de s'assurer que $S C H$ est bien le gène responsable de la NF2, l'ADNc a été utilisé comme sonde dans des analyses de l'ADN des malades par PFGE. Plus de 42 malades NF2 et 40 personnes non atteintes ont ainsi été testés. Deux malades montraient des délétions de 80 et $15 \mathrm{~kb}$ dans la région du gène $S C H$. De même, l'analyse de l'ADN de 60 malades et de 90 sujets témoins réalisée par la technique de Southern a permis d'observer deux autres délétions de $1 \mathrm{~kb}$ chez des personnes atteintes de la NF2 [7]. Voulant connaitre l'effet d'une de ces délétions interstitielles sur l'ARNm correspondant au gène SCH, cet ARN a été sélectionné, par transcription inverse suivie d'une amplification par réaction en chaîne (RT-PCR : reverse transcription - polymerase chain reaction), à partir de la lignée cellulaire dérivée du malade R2142 (figure 2C) et sa séquence déterminée. Les résultats obtenus montrent que les exons 2,3 et 4 sont absents de cette forme anormale d'ARNm provoquant ainsi la synthèse d'une protéine plus courte et, probablement, non fonctionnelle [7]. L'analyse du gène $S C H$ de patients atteints de la NF2 a aussi permis de mettre en évidence des mutations ponctuelles [7]. La majorité des mutations observées conduit à la synthèse d'une protéine tronquée. Certaines de ces mutations présentent une coségrégation avec la maladie chez les patients d'une même famille, suggérant ainsi un lien entre la mutation du gène SCH et la NF2 [7]. Toutefois, l'observation de trois cas 


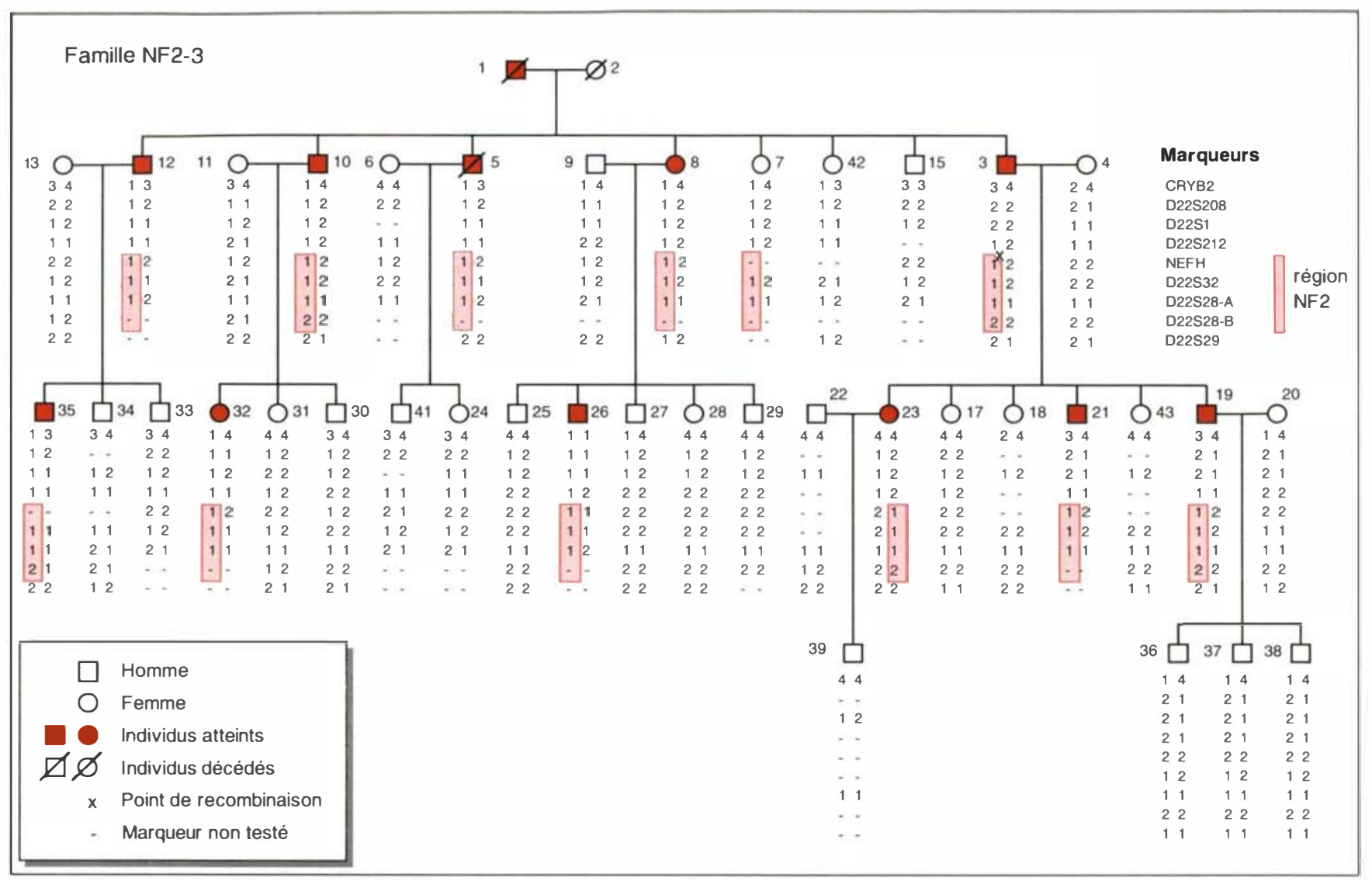

Figure 1. Liaison génétique dans une famille NF2. Plusieurs marqueurs polymorphes sont analysés dans une famille dont plusieurs membres sont atteints de la NF2. Sous chacun des individus on retrouve l'ordre des allèles qu'il possède pour chaque marqueur étudié (haplotype). L'ordre de ces marqueurs sur le chromosome est indiqué dans le coin supérieur droit (centromère vers télomère). A la suite d'une recombinaison homologue, l'haplotype des chromosomes est modifié. La présence de recombinants permet de délimiter la région minimale dans laquelle se trouve le gène recherché. Par exemple, une recombinaison survenue chez l'individu 3, entre les marqueurs D22S212 et NEFH, et transmise à son fils atteint de la NF2 (I'individu 23), permet de situer le gène de cette maladie du côté télomérique du marqueur D22S212. La région du gène NF2 est entre NEFH et D22S32 (portion encadrée de l'haplotype).

de mutations du gène $S C H$ chez des individus atteints, mais dont les parents (non atteints) possèdent chacun deux allèles normaux, est primordiale [7]. L'apparition de ces nouvelles mutations chez des malades n'ayant pas d'antécédents familiaux est considérée comme la preuve du rôle joué par le gène $S C H$ dans la genèse de la NF2 et permet d'affirmer que le gène $\mathrm{SCH}$ est bien le gène $N F 2$.

\section{La schwannomine}

L'ADNc du gène $S C H$ que nous avons isolé contient un cadre ouvert de lecture et code pour une protéine de 66 kDa. Des recherches effectuées dans les banques de données mon$\mathrm{m} / \mathrm{s} n^{\circ} \mathrm{I}$, vol. 11 , janvier 95 trent que la schwannomine est homologue des protéines codées par une famille de gènes comprenant les gènes de l'ezrine (similitude de $45,1 \%)$, de la radixine $(44,9 \%)$ et de la moésine $(45,3 \%)$, un groupe de protéines appartenant à la superfamille de la protéine érythrocytaire bande 4.1 (similitude de $23,3 \%$ avec la schwannomine) $[7,8]$. Les niveaux de similitude augmentent à environ $62 \%$ pour l'ezrine, la radixine et la moésine lorsque seules leurs régions amino-terminales sont considérées (figure 3). Sur la base des études antérieures faites sur les protéines de cette famille, il est possible de formuler l'hypothèse selon laquelle la région amino-terminale de la schwannomine se lierait à un complexe protéique membranaire alors que la partie carboxy-terminale interagirait avec le cytosquelette et, plus particulièrement, l'actine (figure 3). Selon cette hypothèse, la schwannomine pourrait être localisée à la face interne de la membrane cellulaire où elle stabiliserait l'ancrage du cytosquelette à la membrane. Il faut noter qu'au moins un autre produit de gènes impliqués dans la prédisposition génétique aux tumeurs, la protéine APC, aurait une localisation intracellulaire comparable. Récemment, il a été montré que la protéine APC interagit avec les caténines $\beta$ et, peut-être, $a[24,25]$.

Des analyses du profil d'expression de $\mathrm{SCH}$ montrent que des ARNm correspondant à ce gène sont présents dans plusieurs tissus tels que le pou- 


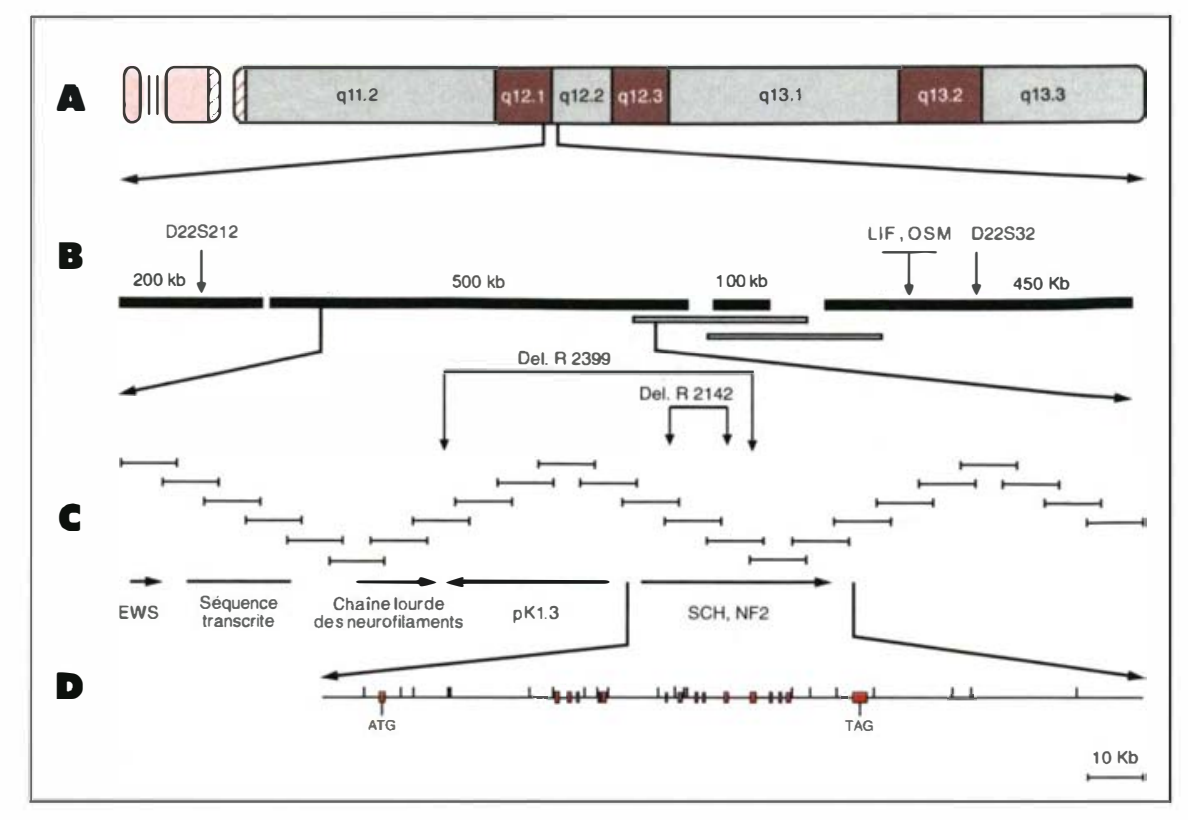

\section{RÉFÉRENCES}

23. Bianchi $\mathrm{AB}$, Hara $\mathrm{T}$, Ramesh $\mathrm{V}$, et al. Mutations in transcript is of orms of the neurofibromatosis 2 gene in multiple human tumour types. Nature (jenet $1994 ; 6$ : 185-92.

24. Rubinfeld B, Souza B, Albert l, el al. Association of the APC; gene product with Bcatenin. Science 1993 ; 262": 1731-4.

25. Su I.K, Vogelstein B, Kinzler KW. Assor ciation of the APC tumor suppressor protein with catenins. Science $1993 ; 262$ : 1734-7.

26. Twist EC, Ruttledge MH, Rousseau M, et al. The neurofibromatosis type 2 gene is inactivated in schwannomas. Hum Mol (jenet $1994 ; 3: 147-51$.

27. Jacoby L.B, Mac Collin M, Louis DN, et al. Exon scamning for mutation of the NF2 gene in schwannomas. Hum Mol (jenet 1994 ; $3: 413-9$.

28. Irving RM, Moffat DA, Hardy DG, Borton DE, Xuereb JH, Maher ER. Somatic NF2 gene mutations in familial and non-familial vestibular schwannoma. Hum Mol (jenet $1994 ; 3: 347-50$

29. Ruttledge MH, Sarrazin J, Rangaratnam $\mathrm{S}$, el al. Evidence for the complete inactivation of the NF2 gene in the majority of sporadic meningiomas. Nature (ienet $1994 ; 6$ : $180-4$

30. Claudio JO, Marineau (;, Rouleau (iA The mouse homologue of the neurotibromatosis type 2 gene is highly conserved. Hum Mol Genet 1994 ; 3 : 185-90.

31. Haase VH, Trofatter JA, Mac Collin M, et al. The murine NF2 homologue encodes a highly conserved merlin protein with alternative forms. Hum Mol Genet 1994 ; 3 : 407-11.

32. Rubio MP, Correa KM, Ramesh V, et al. Analysis of the neurofibromatosis 2 gene in human ependymomas and astrocytomas.

Figure 2. Cartographie physique de la région entourant le gène de la neurofibromatose de type 2. La figure est divisée en 4 parties $(A$ à $D)$ et présente une résolution croissante. 2A: Représentation schématique du chromosome 22 humain. La longueur de son bras long est estimée à 40 millions de paires de bases. Le centromère est situé sur la gauche. 2B : Carte de la région comprise entre les loci D22S212 et D22S32 situés de part et d'autre du gène NF2. Chaque rectangle noir correspond à des contigs de cosmides. Les deux rectangles blancs représentent deux chromosomes artificiels de levure (YAC) qui permettent de couvrir deux régions pour lesquelles des cosmides n'avaient pu être obtenus. Du côté centromérique, la distance séparant le contig de $200 \mathrm{~kb}$ de celui de $500 \mathrm{~kb}$ est inférieure à 0,5 kb. A proximité du locus D22S32 sont localisés les gènes codant pour l'oncostatine M (OSM) et le facteur inhibant la leucémie (LIF), deux cytokines étroitement apparentées. 2C: Détail d'une partie du contig de cosmides de $500 \mathrm{~kb}$. Chez les malades atteints de NF2, R2399 et $R 2142$, cette région est le siège de délétions ayant permis de cibler précisément la recherche du gène NF2. A ce jour, cinq gènes ont pu être identifiés sur ce contig. Du centromère vers le télomère, il s'agit de EWS, gène systématiquement réarrangé par translocation chromosomique dans la famille des tumeurs d'Ewing; une région transcrite peu caractérisée (transcrit de $4 \mathrm{~kb}$ ); le gène codant pour la chaîne lourde des neurofilaments (NEFH); le gène pK1.3 dont la séquence de l'ADNc ne présente aucune ressemblance avec des gènes connus; enfin $\mathrm{SCH}$, le gène de la neurofibromatose de type 2. 2D: Structure génomique du gène SCH/NF2. Ce gène comprend 16 exons (rectangles rouges) qui sont répartis sur un total d'environ $120 \mathrm{~kb}$.

mon, le rein, les ovaires, le sein, le placenta et les neuroblastes [7]. Le plus fort niveau d'expression a été observé dans le cerveau fotal. Ces résultats montrent que le gène $S C H$ est largement exprimé et que cette expression semble varier durant le développement. De plus, un ARNm correspondant à un épissage alternatif (isoforme II) montre un profil d'expression différent de celui de la forme originale (isoforme I) [23]. Cette large expression du gène $\mathrm{SCH}$ est inattendue puisque l'essentiel des tu- meurs observées chez les patients NF2 sont celles dérivées des cellules de Schwann et des méninges.

\section{Mutations du gène SCH}

$\mathrm{Si}$, comme il était postulé, le gène $N F 2$ est inactivé par un processus en deux étapes (hypothèse de Knudson), chacune inactivant un des allèles, il devrait être possible de trouver des mutations de $S C H$ dans les schwannomes et les méningiomes se développant chez des sujets n'étant 


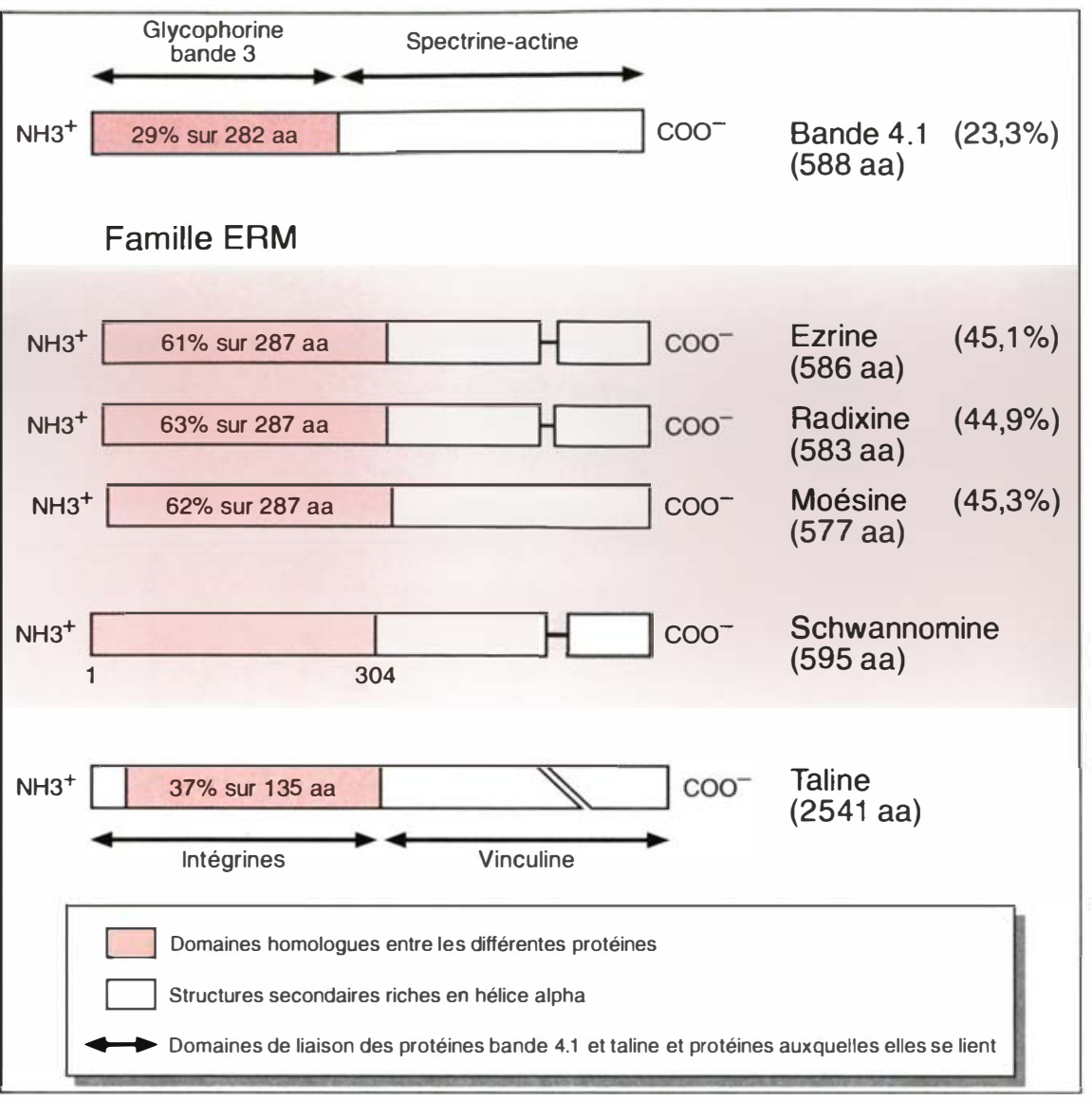

Figure 3. Homologie des protéines ERM. Homologie des protéines humaines bande 4.1, ezrine, radixine, moésine et taline avec la schwannomine humaine. Les régions rosées correspondent aux régions de plus forte identité entre les différentes protéines. Les pourcentages d'identité de chaque protéine avec les acides aminés 1 à 304 de la schwannomine sont indiqués dans les cases rosées. Les domaines d'interaction caractérisés de la bande 4.1 et de la taline sont indiqués par des flèches. Les protéines interagissant avec ces domaines sont associées aux flèches. La taille des protéines est indiquée en nombre d'acides aminés (aa).

pas atteints de NF2. Une telle recherche fut effectuée par plusieurs groupes utilisant des techniques différentes telles que la détection de polymorphismes de conformation de l'ADN simple brin (SSCP: single strand conformational polymorphism), l'analyse par électrophorèse sur gradient de gel dénaturant (DGGE: denaturing gradient gel electrophoresis) et la RT-PCR.

Les schwannomes sporadiques sont les tumeurs les plus étudiées à ce jour. L'analyse de 183 tumeurs a permis de détecter 50 mutations du gène SCH (Tableau II). L'introduction de codons stop, les déphasages du cadre de lecture ainsi que les erreurs d'épissage conduisent à la synthèse de protéines tronquées probable$m / s n^{\circ} 1$, vol. 11 , jonvier 95 ment, à la synthèse d'une protéine tronquée (Tableau II). Fait intéressant, Ruttledge et al. rapportent que toutes les mutations observées proviennent de tumeurs où existe une monosomie complète ou partielle du chromosome $22\left(\mathrm{~m} / \mathrm{s}{ }^{\circ} 6-7\right.$, vol. 10 , $p$. 729 [29]). Ce type tumoral avec monosomie du chromosome 22 représente environ $60 \%$ des méningiomes. Ces résultats laissent supposer que, pour environ $40 \%$ des méningiomes, le gène $S C H$ ne serait pas impliqué. L'observation de l'apparition fréquente de mutations ponctuelles et de délétions alléliques au sein de tumeurs sporadiques ou se développant chez les malades atteints de la NF2 confirme l'hypothèse que le gène $S C H$ joue un rôle "suppresseur de tumeur " à l'intérieur des cellules de Schwann et des cellules des méninges.

La présence occasionnelle d'épendymomes et de gliomes chez les malades NF2 laisse supposer que le gène SCH pourrait jouer un rôle dans la genèse de ces tumeurs. De même, il existe une série de tumeurs dans lesquelles une monosomie du chromosome 22 est souvent observée : les phéochromocytomes et les carcinomes du sein et du côlon appartiennent à ce groupe. La monosomie du chromosome 22 rend plausible l'implication du gène $S C H$ dans le développement de ces tumeurs. Bien que les travaux soient préliminaires, les résultats obtenus à ce jour ne montrent l'implication du gène $\mathrm{SCH}$ que dans un épendymome et un carcinome du sein (Tableau II). Aucune mutation n'a été observée dans les gliomes, les phéochromocytomes et les carcinomes du côlon, des tumeurs susceptibles d'être causées par un défaut du gène $N F 2$. Toutefois, nous ne pouvons exclure que le gène $\mathrm{SCH}$ ne soit pas éventuellement impliqué dans ces types de tumeur. Ainsi, des mutations du gène $S C H$ ont été mises en évidence dans des mélanomes malins, des tumeurs non reliées à la NF2 et ne montrant pas de monosomie fréquente du chromosome 22 .

\section{Perspectives}

Ces résultats suggèrent que le gène $N F 2$ est surtout impliqué dans le développement des schwannomes et des méningiomes. Ces tumeurs représen- 


\begin{tabular}{|c|c|c|c|c|}
\hline \multicolumn{5}{|c|}{$\begin{array}{l}\text { Tableau II } \\
\text { MUTATIONS DU GĖNE SCH DANS DIFFÉRENTS TYPES DE TUMEURS }\end{array}$} \\
\hline $\begin{array}{l}\text { Type de } \\
\text { tumeurs }\end{array}$ & $\begin{array}{l}\text { Nombre de } \\
\text { tumeurs } \\
\text { testées }\end{array}$ & $\begin{array}{l}\text { Nombre de } \\
\text { mutations } \\
\text { observées }\end{array}$ & Type de mutations & Références \\
\hline \multicolumn{5}{|l|}{ Tumeurs sporadiques } \\
\hline Schwannomes & 183 & 50 & $\begin{array}{l}\text { 43/50 déphasages du cadre de lecture, codon STOP } \\
5 / 50 \text { délétions internes } \\
2 / 50 \text { substitutions d'acide aminé }\end{array}$ & [26-28] \\
\hline Méningiomes & 181 & 26 & 26/26 déphasages du cadre de lecture, codon STOP & {$[7,29]$} \\
\hline Gliomes & 30 & 0 & & [32] \\
\hline Mélanomes & 20 & 6 & $6 / 6$ délétions internes & [23] \\
\hline $\begin{array}{l}\text { Carcinome } \\
\text { du côlon }\end{array}$ & 20 & 0 & & [23] \\
\hline $\begin{array}{l}\text { Carcinome } \\
\text { du sein }\end{array}$ & 14 & 1 & 1/1 délétion interne & [23] \\
\hline Épendymomes & 8 & 1 & 1/1 déphasage du cadre de lecture, codon STOP & [32] \\
\hline Phéochromocytomes & 5 & 0 & & [23] \\
\hline \multicolumn{5}{|c|}{ Tumeurs de patients NF2 } \\
\hline Schwannomes & 26 & 16 & $\begin{array}{l}\text { 13/16 déphasages du cadre de lecture, codon STOP } \\
2 / 16 \text { délétions internes } \\
1 / 16 \text { substitution d'acide aminé }\end{array}$ & {$[3,26-28]$} \\
\hline
\end{tabular}

tent une proportion importante des tumeurs nerveuses et donc, bien que la NF2 soit une maladie héréditaire rare, des altérations somatiques de son gène peuvent conduire relativement fréquemment à des tumeurs. Bien que le gène $N F 2$ semble avoir une expression relativement ubiquitaire, nous n'observons ses altérations que dans un spectre étroit de types tumoraux. Comme il est peu vraisemblable que des altérations génomiques affectant le gène $N F 2$ ne puissent se produire que dans les cellules progénitrices de ces tumeurs, il serait intéressant de comprendre pourquoi d'autres types tumoraux ne sont pas retrouvés. Deux groupes ont récemment rapporté le clonage de l'ADNc $N F 2$ de souris $[30,31]$. Ce gène est très similaire au gène humain et devrait permettre la mise au point d'un modèle animal de la NF2. Un tel modèle est réalisable en inactivant, par recombinaison homologue, le gène possible d'examiner si ces souris, à l'état hétérozygote pour le gène inactivé, présentent une prédisposition à développer des schwannomes et des méningiomes. Les con-séquences sur l'embryogenèse et le développement des souris ne possédant pas de schwannomine fonctionnelle pourront aussi être précisées.

Aujourd'hui, le rôle fonctionnel de la schwannomine peut aussi être précisé par des approches biochimiques et cellulaires consistant à examiner l'expression tissulaire, la localisation subcellulaire et les interactions de la protéine grâce au développement d'anticorps spécifiques. L'examen du rôle "suppresseur de tumeur» de la schwannomine par réintroduction d'un gène fonctionnel dans des cellules tumorales se heurte actuellement au fait que les schwannomes et les méningiomes sont des tumeurs bénignes et qu'il n'a pas été possible jusqu'à présent d'établir des lignées en culture à partir de ces tumeurs

\section{Summary}

The neurofibromatosis type 2 gene

Neurofibromatosis type 2 (NF2) is a genetic disease predisposing to the development of vestibular schwannomas and meningiomas. Although NF2 is a rare disorder, sporadic schwannomas and meningiomas represent an important fraction of all brain tumours. Using a positional cloning strategy, it was possible to identify on chromosome 22 a gene that is most probably inactivated in the majority of NF2 affected individuals. This gene codes for a $66 \mathrm{kDa}$ protein called schwannomin or merlin which shows high sequence homology to proteins of the ezrin-radixin-moesin (ERM) family. By analogy to proteins of this family, we hypothesize that schwannomin is involved in the organization of membrane cytoskeleton junctions. Cytogenetic studies and mutations analysis of tumour DNA suggest that schwannomin acts as a tumour suppressor gene. 